PAPER

\title{
Prognostic significance of admission levels of troponin I in patients with acute ischaemic stroke
}

\author{
E Di Angelantonio, M Fiorelli, D Toni, M L Sacchetti, S Lorenzano, A Falcou, M V Ciarla, M Suppa, \\ L Bonanni, G Bertazzoni, F Aguglia, C Argentino
}

J Neurol Neurosurg Psychiatry 2005;76:76-81. doi: 10.1136/jnnp.2004.041491

See end of article for authors' affiliations

Correspondence to:

Correspondence to:
Dr M Fiorelli, Department of Neurological Sciences, University of Rome "La Sapienza", Viale dell'Università 30, 00185 Rome, Italy; marco fiorelli@uniromal.it

Received 13 March 2004 In revised form 8 April 2004

Accepted 21 April 2004

\begin{abstract}
Objectives: Successful prediction of cardiac complications early in the course of acute ischaemic stroke could have an impact on the clinical management. Markers of myocardial injury on admission deserve investigation as potential predictors of poor outcome from stroke.

Methods: We prospectively investigated 330 consecutive patients with acute ischaemic stroke admitted to our emergency department based stroke unit. We analysed the association of baseline levels of cardiac troponin I (cTnl) with (a) all-cause mortality over a six month follow up, and (b) inhospital death or major non-fatal cardiac event (angina, myocardial infarction, or heart failure).

Results: cTnl levels on admission were normal (lower than $0.10 \mathrm{ng} / \mathrm{ml}$ ) in 277 patients $(83.9 \%)$, low positive $(0.10-0.39 \mathrm{ng} / \mathrm{ml})$ in $35(10.6 \%)$, and high positive $(0.40 \mathrm{ng} / \mathrm{ml}$ or higher) in $18(5.5 \%)$. Six month survival decreased significantly across the three groups ( $<<0.0001$, log rank test for trend). On multivariate analysis, cTnl level was an independent predictor of mortality (low positive cTnl, hazard ratio (HR) $2.14 ; 95 \% \mathrm{Cl} 1.13$ to $4.05 ; \mathrm{p}=0.01$; and high positive $\mathrm{cTnl}, \mathrm{HR} 2.47 ; 95 \% \mathrm{Cl} 1.22$ to 5.02 ; $p=0.01$ ), together with age and stroke severity. cTnl also predicted a higher risk of the combined endpoint "inhospital death or non-fatal cardiac event". Neither the adjustment for other potential confounders nor the adjustment for ECG changes and levels of CK-MB and myoglobin on admission altered these results. Conclusions: cTnl positivity on admission is an independent prognostic predictor in acute ischaemic stroke. Whether further evaluation and treatment of cTnl positive patients can reduce cardiac morbidity and mortality should be the focus of future research.
\end{abstract}

study. Patients are admitted to this six bed unit from the emergency room at the discretion of the neurologist on duty, after computed tomography (CT) or magnetic resonance imaging (MRI) has confirmed the diagnosis of acute stroke. There are no selection criteria for referral to the SU. However, in the event that more patients with stroke present simultaneously to the emergency department than are beds available in the SU, priority is given to patients with more recent onset.

The study protocol was approved by the institutional review board. The requirement for informed consent was waived because screening for myocardial injury is usual practice in patients with acute cardiovascular conditions.

\section{Baseline data collection}

On admission to the SU, a venous blood sample was obtained for cardiac markers; the levels of cTnI were measured by immunoassay using a Dimension RxL system (Dade Behring; Deerfield, IL) in the hospital central laboratory on receipt of the sample. According to the manufacturer this system can detect a minimum cTnI level of $0.04 \mathrm{ng} / \mathrm{ml}$, while the 99th percentile of the normal reference range is $0.07 \mathrm{ng} / \mathrm{ml}$. The system allows CK-MB mass and myoglobin levels to be assayed simultaneously on the same sample. This was done in most cases.

Other data collected on admission included age, sex, history of the disease, presence of vascular risk factors, and findings at general physical examination, including the Killip class. Killip class I was defined as the absence of rales over the lung fields and the absence of S3 gallop. Class II included

Abbreviations: cTnl, cardiac troponin I; cTnT, cardiac troponin T; MI, myocardial infarction; NIHSS, National Institutes of Health Stroke Scale; SU, stroke unit 
Table 1 Baseline characteristics and medical history of the patients by troponin levels

\begin{tabular}{|c|c|c|c|c|c|}
\hline \multirow[b]{2}{*}{ Characteristics } & \multicolumn{3}{|l|}{ cTnl (ng/ml) } & \multirow[b]{2}{*}{$p$ linear } & \multirow[b]{2}{*}{$p$ value } \\
\hline & $<0.1$ & $0.1-0.39$ & $\geqslant 0.4$ & & \\
\hline No. of patients* & $277 / 330$ (83.9) & $35 / 330(10.6)$ & $18 / 330(5.5)$ & & \\
\hline \multicolumn{6}{|l|}{ Demographics } \\
\hline Age in years $\dagger$ & $68.5(12.4)$ & $72.9(13.0)$ & $69.7(14.2)$ & 0.688 & 0.148 \\
\hline Men* & $140 / 277(50.5)$ & $19 / 35(54.3)$ & $11 / 18(61.1)$ & 0.356 & 0.645 \\
\hline Women* & $137 / 277$ (49.5) & $16 / 35(45.7)$ & $7 / 18(38.9)$ & 0.356 & 0.645 \\
\hline \multicolumn{6}{|l|}{ Medical history* } \\
\hline Hypertension & $173 / 275$ (62.9) & $24 / 35(68.6)$ & $12 / 18(66.7)$ & 0.549 & 0.778 \\
\hline Diabetes mellitus & $55 / 275(20.0)$ & $4 / 35(11.4)$ & $5 / 18(27.8)$ & 0.969 & 0.320 \\
\hline Atrial fibrillation & $60 / 276(21.7)$ & $14 / 35(40.0)$ & $3 / 18$ (16.7) & 0.405 & 0.044 \\
\hline Prior myocardial infarction & $36 / 275(13.1)$ & $6 / 35(17.1)$ & $4 / 18(22.2)$ & 0.225 & 0.476 \\
\hline Stable angina & $9 / 275(3.3)$ & - & - & 0.214 & 0.410 \\
\hline Prior stroke & $47 / 277(17.0)$ & $13 / 35(37.1)$ & $4 / 18(22.2)$ & 0.113 & 0.017 \\
\hline Prior transient ischaemic attack & $33 / 277$ (11.9) & $3 / 35(8.6)$ & - & 0.057 & 0.261 \\
\hline Current smoker & $68 / 273(24.9)$ & $3 / 34(8.8)$ & $6 / 17$ (35.3) & 0.771 & 0.060 \\
\hline Hypercholesterolaemia & $45 / 273(16.5)$ & $5 / 33(15.2)$ & $1 / 18(5.6)$ & 0.265 & 0.465 \\
\hline \multicolumn{6}{|l|}{ Physical exam } \\
\hline Heart rate, bpm & $77(15)$ & $84(18)$ & $85(23)$ & 0.033 & 0.006 \\
\hline Systolic blood pressure, $\mathrm{mm} \mathrm{Hg \dagger}$ & $154(27)$ & $160(43)$ & $160(32)$ & 0.500 & 0.532 \\
\hline Diastolic blood pressure, $\mathrm{mm} \mathrm{Hg \dagger}$ & $90(16)$ & $93(23)$ & $90(15)$ & 0.914 & 0.705 \\
\hline Killip class $\geqslant \|^{*}$ & $25 / 277(9.0)$ & $8 / 35(22.9)$ & $8 / 18(44.4)$ & $<0.001$ & $<0.001$ \\
\hline Interval between stroke onset and cTnl assay $<6$ hours* & $137 / 277(49.5)$ & $15 / 35(42.9)$ & $8 / 18(44.4)$ & 0.475 & 0.717 \\
\hline NIHSS score† & $6.9(6.5)$ & $12.4(9.8)$ & $13.8(7.9)$ & $<0.001$ & $<0.001$ \\
\hline
\end{tabular}

patients who had rales across $\leqslant 50 \%$ of the lung fields or the presence of an S3 gallop. Class III was defined as rales across $>50 \%$ of the lung fields and class IV as pulmonary oedema with hypoperfusion (cardiogenic shock). The neurological deficit was graded using the National Institutes of Health Stroke Scale (NIHSS). ${ }^{8}$ The aetiology of the stroke was classified according to the Trial of ORG 10172 in Acute Stroke Treatment (TOAST) criteria. ${ }^{9}$

All patients had a standard 12 lead ECG on admission recorded at $25 \mathrm{~mm} / \mathrm{s}$ and calibrated at $1 \mathrm{~cm} / \mathrm{mV}$. For the purposes of this study, ECG tracings were coded and interpreted by two investigators blinded to the rest of the patients' data, who analysed heart rate and rhythm, duration of PR, QT and rate-corrected QT (QTc by Bazzett's formula), duration, axis, and morphology of the QRS complex, degree and pattern of ST segment displacement, T wave configuration, and the Sokolow-Lyon voltage. Interobserver differences were resolved by consensus. P wave morphology and atrioventricular conduction were not evaluated in patients with atrial fibrillation, atrial flutter, and in those with pacemakers.

\section{Follow up and endpoints}

Incident inhospital events were ascertained and characterised by structured data abstraction from medical records. Patients who survived the acute phase and were discharged home or to an inpatient rehabilitation, or a nursing home were further followed up at minimum six months after the index stroke by telephone calls. The patient themselves, or alternatively a family member or a caregiver, were interviewed by a trained assessor using an ad hoc questionnaire that included the occurrence and date of death after hospital discharge as a specific item.

Mortality was the main endpoint of the study. The detailed information available regarding the period of hospitalisation was used to classify inhospital death as due to neurological or cardiac causes following slightly modified published criteria. ${ }^{10}$ Inhospital deaths from other causes were not observed in this cohort. Death was "neurological" if due to the incident stroke or recurrent stroke and it was considered as related to the incident stroke in the following cases: $(a)$ death due to stroke induced brain herniation (progressive stroke, brain oedema, intra-infarct haematoma) or respiratory arrest (brain stem infarct); (b) death from aspiration or aspiration pneumonia due to dysphagia in the acute stroke phase; and (c) death by incident stroke related immobilisation (for example, pneumonia or pulmonary embolism). "Cardiac" deaths were due to acute myocardial infarction (MI), heart failure, or arrhythmia (including sudden death). We also examined the combined endpoints of inhospital death or non-fatal MI, and inhospital death or one of the acute nonfatal cardiac events: MI or heart failure. MI was defined according to the guidelines of the European Society of Cardiology. ${ }^{11}$

When a patient had more than one of these events, only the first was considered in the analysis. All clinical and instrumental cardiac variables were defined according to recently published guidelines. ${ }^{12}$

\section{Data analysis}

Patients were categorised into one of three groups according to the level of cTnI on admission: normal (lower than $0.10 \mathrm{ng} / \mathrm{ml})$, low positive $(0.10-0.39 \mathrm{ng} / \mathrm{ml})$, and high positive $(0.40 \mathrm{ng} / \mathrm{ml}$ or higher). These cut points were chosen on the basis of the most recent relevant literature. ${ }^{13}{ }^{14}$ The groups were compared with regard to the admission variables and follow up events using $\chi^{2}$ test for trend, and one way analysis of variance (ANOVA) for linear trend. Pearson's $\chi^{2}$, conventional one way ANOVA, and Wilcoxon's signed rank test were also performed as ancillary statistics. Unadjusted KaplanMeier survival plots were compared using the log rank test for linear trend.

Cox proportional hazards models were used to compute hazard ratios of all-cause mortality according to cTnI levels after adjustment for potential confounders. Patients with normal cTnI served as the reference group. Initial analyses involved adjustments for age, sex, and NIHSS score. Further procedures were conducted to account for the possible influence of any baseline variable associated with cTnI in the univariate analysis at a probability threshold of 0.1 or lower. We also investigated whether the relationship between cTnI level and risk of death was modified when ECG findings 


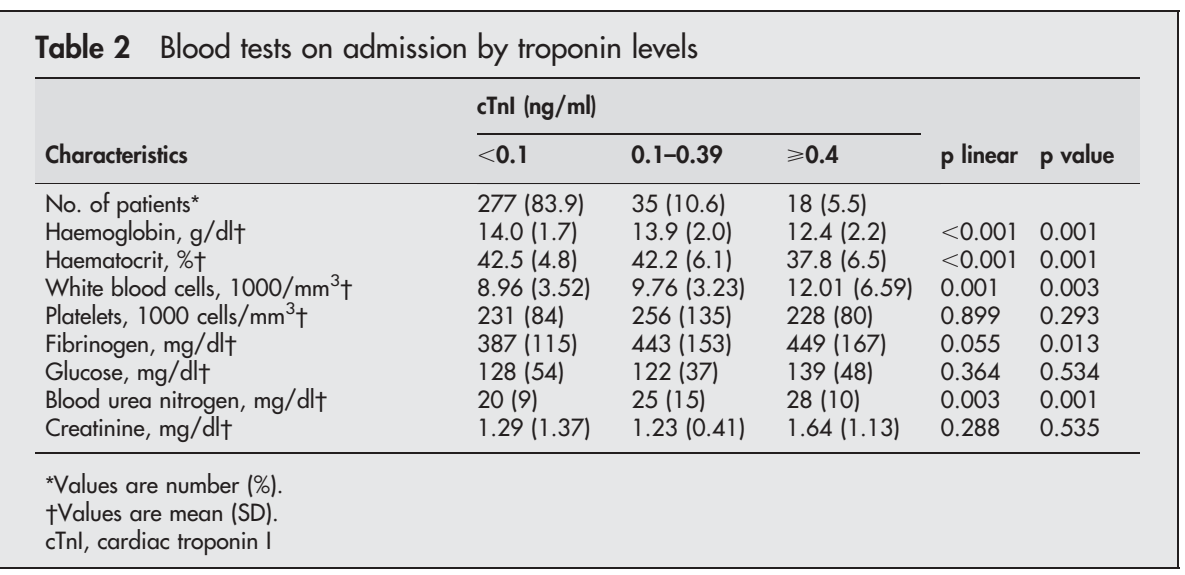

and levels of CK-MB and myoglobin were included in the model. We repeated adjusted analyses within strata of covariates to explore possible effect modification. SPSS statistical software was used for all analyses. A probability value of $<0.05$ was considered statistically significant.

\section{RESULTS}

A total of 330 consecutive patients with acute ischaemic stroke $(48.5 \%$ women, mean age 69 years, $48.5 \%$ admitted within six hours of onset) were included in the study. cTnI levels were normal in $277(83.9 \%)$ patients, low abnormal in $35(10.6 \%)$, and high abnormal in $18(5.5 \%)$. Among the latter, the maximum observed value was $23 \mathrm{ng} / \mathrm{ml}$ and the median was $0.65 \mathrm{ng} / \mathrm{ml}$.

The distribution of ischaemic stroke subtypes was as follows: large vessel disease 79/330 (24\%), small vessel disease $27 / 330(8.2 \%)$, cardioembolic $82 / 330(24.8 \%)$, stroke of undetermined aetiology $125 / 330(37.8 \%)$, and other determined aetiology $17 / 330(5.2 \%)$. There were no significant differences in the distribution of abnormal cTnI levels between the different stroke subtypes.

\section{Physical examination, neurological status, blood tests, and vascular risk factors}

Table 1 gives the findings at clinical examination and from the medical history on admission by cTnI levels. There were no significant differences between the three cTnI groups with regard to age, sex, and delay between stroke onset and assay of markers of myocardial necrosis. Increasing levels of cTnI were associated with an increasingly severe neurological deficit, as measured by the NIHSS score $(p<0.001)$. A significant, positive dose-response relationship was also observed between cTnI and heart rate $(p=0.033)$, and between cTnI and Killip class $\geqslant$ II $(p<0.001)$. Several routine blood tests were significantly associated with the cTnI levels (table 2). In the trend analysis, haemoglobin and haematocrit appeared to decrease ( $\mathrm{p}<0.001$ for both), whereas blood urea nitrogen, white blood cell count and, marginally, fibrinogen, increased $(\mathrm{p}=0.003, \mathrm{p}=0.001$, and $\mathrm{p}=0.055$, respectively) across the three cTnI groups.

\section{CK-MB and myoglobin}

CK-MB levels were available for 300 patients (91\%), and myoglobin levels for 313 (95\%). The upper limits of the reference intervals for CK-MB and myoglobin were $5 \mathrm{ng} / \mathrm{ml}$ and $90 \mathrm{ng} / \mathrm{ml}$, respectively. Among patients with normal, low positive, and high positive cTnI, prevalence rates of abnormally high CK-MB (>5 ng/ml) were 3.2\% $(\mathrm{n}=8), 9.4 \%$ $(n=3)$, and $43.8 \%(n=7)$, respectively $(p=0.001)$ and of abnormally high myoglobin $(>90 \mathrm{ng} / \mathrm{ml})$ were $28.1 \%$ $(\mathrm{n}=74), 52.9 \%(\mathrm{n}=18)$, and $56.3 \%(\mathrm{n}=9)$, respectively $(\mathrm{p}=0.001)$.

\section{ECG findings}

ECGs of 325 (98\%) patients were available for diagnostic coding (table 3$)$. Of these, $14(4 \%)$ had a cardiac pacemaker, 11 in the group with normal cTnI and 3 in the group with high positive cTnI $(\mathrm{p}=0.016)$. These patients were excluded from subsequent analyses. Compared with patients with normal cTnI, patients with low positive, but not those with

Table 3 ECG findings on admission by troponin levels

\begin{tabular}{|c|c|c|c|c|c|}
\hline \multirow[b]{2}{*}{ Characteristics } & \multicolumn{3}{|l|}{ cTnl (ng/ml) } & \multirow[b]{2}{*}{$p$ linear } & \multirow[b]{2}{*}{$\mathbf{p}$} \\
\hline & $<0.1$ & $0.1-0.39$ & $\geqslant 0.4$ & & \\
\hline No. of patients* & $273 / 325$ (84.0) & $34 / 325(10.5)$ & $18 / 325(5.5)$ & & \\
\hline Pacemaker* & $11 / 273(4.0)$ & - & $3 / 18(16.7)$ & 0.124 & 0.016 \\
\hline Atrial fibrillation or flutter* & $49 / 273(17.9)$ & $13 / 34(38.2)$ & $2 / 18(11.1)$ & 0.397 & 0.013 \\
\hline PR, mst‡ & $168(28)$ & $177(33)$ & $166(29)$ & 0.789 & 0.350 \\
\hline QRS duration, ms†§ & $102(18)$ & $102(18)$ & $104(56)$ & 0.915 & 0.993 \\
\hline QTc, ms†§ & $394(46)$ & $396(56)$ & 415 (59) & 0.102 & 0.260 \\
\hline ST-T depression*§ & $11 / 261(4.2)$ & $3 / 34(8.3)$ & $3 / 15(20.0)$ & 0.007 & 0.022 \\
\hline ST--T elevation*§ & $2 / 261(0.8)$ & $1 / 34(2.9)$ & - & 0.520 & 0.439 \\
\hline T wave inversion* $\S$ & $49 / 261(18.8)$ & $11 / 34(32.4)$ & $6 / 15(40.0)$ & 0.011 & 0.037 \\
\hline$Q$ waves* $\S$ & $34 / 261(13.0)$ & $4 / 34(12.1)$ & $4 / 15(26.7)$ & 0.259 & 0.311 \\
\hline Right bundle branch block*§ & $32 / 262(12.2)$ & $5 / 34(14.7)$ & $3 / 15(20.0)$ & 0.358 & 0.643 \\
\hline Left bundle branch block*§ & $3 / 262(1.1)$ & $1 / 34(2.9)$ & $1 / 15(6.7)$ & 0.082 & 0.206 \\
\hline Sokolow-Lyon index $>35 \mathrm{~mm}^{*} \S$ & $28 / 262(9.9)$ & $5 / 34(14.7)$ & $4 / 15(26.7)$ & 0.042 & 0.109 \\
\hline
\end{tabular}

*Values are number (\%).

†Values are mean (SD).

\pm Patients with atrial fibrillation or flutter, or pacemaker excluded.

§atients with pacemaker excluded.

cTnl, cardiac troponin I. 
Table 4 Inhospital events by troponin levels

\begin{tabular}{|c|c|c|c|c|c|}
\hline \multirow[b]{2}{*}{ Characteristics } & \multicolumn{3}{|l|}{ cTnl (ng/ml) } & \multirow[b]{2}{*}{ p linear } & \multirow[b]{2}{*}{$\mathbf{p}$} \\
\hline & $<0.1$ & $0.1-0.39$ & $\geqslant 0.4$ & & \\
\hline $\begin{array}{l}\text { No. of patients* } \\
\text { Inhospital events (\%) }\end{array}$ & $277 / 330(83.9)$ & $35 / 330(10.6)$ & $18 / 330(5.5)$ & & \\
\hline Death & $20 / 277(7.2)$ & $13 / 35(37.1)$ & $8 / 18(44.4)$ & $<0.001$ & $<0.001$ \\
\hline Death or non-fatal myocardial infarction & $23 / 273(8.4)$ & $14 / 34(41.2)$ & $9 / 17$ (52.9) & $<0.001$ & $<0.001$ \\
\hline Death or any non-fatal cardiac event & $38 / 273$ (13.9) & $15 / 34(44.1)$ & $9 / 17(52.9)$ & $<0.001$ & $<0.001$ \\
\hline
\end{tabular}

high positive cTnI, more frequently had atrial fibrillation or flutter. Among the whole cohort, prevalence of ECG findings related to myocardial ischaemia or necrosis was $1 \%(3 / 310)$ for ST segment elevations, $5.4 \%$ (17/310) for ST segment depressions, $21.3 \%$ (66/310) for T wave inversions, and 13.5\% $(42 / 310)$ for Q waves. ST segment depression and T wave inversion were significantly more likely with increasing cTnI levels $(p=0.007$ and $p=0.011$, respectively $)$. However, prevalence of the latter ECG abnormalities in the presence of abnormal troponin levels was modest, as in the group with high positive CTnI only $40 \%$ of patients had a $\mathrm{T}$ wave inversion, and only $20 \%$ had ST segment depression. In the same group, cTnI levels did not differ between patients with at least one abnormal ECG finding compatible with ischaemia or necrosis as opposed to those with an unremarkable tracing $(p=0.25)$. Finally, ventricular hypertrophy (Sokolow-Lyon index) was significantly more likely with increasing cTnI levels.

\section{Outcome}

All patients had a complete inhospital follow up; two patients ( $0.6 \%$ of the initial cohort) could not be reached for post discharge telephone interviews. The remainder had a follow up of at least six months (mean 152 days, median 144, range 2-489), during which 65 (19.7\%) patients died, 41 in hospital and 24 after discharge. There were 14 cardiac deaths (eight MI, five heart failure, one sudden death), which in the three cTnI groups accounted for $25.0 \%, 30.8 \%$, and $62.5 \%$, respectively, of the inhospital deaths $(\mathrm{p}=0.1)$. During hospital stay five $(1.5 \%)$ patients had a non-fatal MI, and $12(3.6 \%)$ another major non-fatal cardiopulmonary event. Rates of death, death or non-fatal MI, and death or major non-fatal cardiopulmonary event during hospital stay are summarised in table 4. All these endpoints were more common in patients with low or high abnormal cTnI, showing a graded trend. In particular, the inhospital casefatality ratio was $7.2 \%(20 / 277)$ in patients with cTnI $<0.10 \mathrm{ng} / \mathrm{ml}, 37.1 \%(31 / 35)$ in patients with cTnI 0.10 $0.39 \mathrm{ng} / \mathrm{ml}$, and $44.4 \%(8 / 18)$ in those with cTnI $\geqslant 0.40 \mathrm{ng} / \mathrm{ml}$ $(p<0.001)$. Patients with elevated cTnI were mostly treated with nitrates, in some cases with the addition of $\beta$-blockers. They were all also on aspirin, or low molecular weight heparin, or both. No invasive diagnostic or therapeutic interventions (coronary angiography, angioplasty, thrombolysis) were performed.

The Kaplan-Meier curves in the fig 1 show that there was a distinctive prognostic gradient across the three cTnI groups over the entire follow up $(\mathrm{p}<0.0001)$, with mortality increasing with cTnI levels. Table 5 gives the hazard ratios for all-cause mortality in patients with abnormal troponin levels. cTnI was still an independent predictor of mortality after adjustment of age and baseline NIHSS score. Neither the additional control for ECG abnormalities, CK-MB, and myoglobin, nor that for possible confounders (any other clinical or instrumental baseline variable associated with cTnI at univariate analysis at a probability threshold of 0.1 ) materially altered these results.

\section{DISCUSSION}

The release of cardiac enzymes in the acute phase of ischaemic stroke began to be documented in the late $1970 s .{ }^{15}$ On the basis of further research, myocardial injury after stroke or other acute cerebral lesions was attributed to abnormally high levels of plasma catecholamines secondary to rapidly increasing intracranial pressure. ${ }^{16}$ Currently, cardiac troponins are considered the most accurate biomarker of myocardial injury available in the clinical setting. In the first report of an association between troponin levels and poor outcome in a cohort of consecutive ischaemic stroke patients, ${ }^{6}$ the criterion for abnormally high troponin was a concentration of cTnT higher than $0.1 \mu \mathrm{g} / \mathrm{l}$. Assays were performed 12-72 hours after admission and after an unspecified delay from the onset of stroke. Troponin levels were elevated in $17 \%$ of the patients, and troponin positivity was found to be associated with a threefold increase in risk of inhospital death. Further evidence of a link between high troponin levels and poor prognosis came from a cohort of patients hospitalised for an acute disease of the central nervous system. In the whole series, patients with cTnI of $0.4 \mathrm{ng} / \mathrm{ml}$ or higher had an odds ratio of 6.4 for 30 day mortality. ${ }^{7}$ However, the cohort included only six patients with ischaemic stroke.

Overall, at the threshold of CTnI $\geqslant 0.1 \mathrm{ng} / \mathrm{ml}, 16.6 \%$ of our patients tested positive for abnormal troponin on admission. Using the more conservative cut point of $\mathrm{cTnI} \geqslant 0.4 \mathrm{ng} / \mathrm{ml}$, $5.5 \%$ tested positive. CK-MB and myoglobin showed a consistent association with troponin levels but, compared with the latter, confirmed to be less accurate indicators of myocardial damage. Taking a cTnI concentration $\geqslant 0.4 \mathrm{ng} / \mathrm{ml}$ as the reference standard, CK-MB appeared to have low sensitivity $(43.8 \%)$, but good specificity $(96.8 \%)$, whereas myoglobin had slightly higher sensitivity $(56.0 \%)$ and worse specificity $(71.9 \%)$. These findings indicate, on average, myocardial injury was slight in our cohort, and it would have escaped detection in approximately half of the cases if the admission work up had been limited to CK-MB and myoglobin. ECG was not more informative. The tracings on admission were unremarkable in more than $50 \%$ of patients with cTnI levels of $0.4 \mathrm{ng} / \mathrm{ml}$ or higher, and in nearly $80 \%$ of those with cTnI levels between $0.1 \mathrm{ng} / \mathrm{ml}$ and $0.4 \mathrm{ng} / \mathrm{ml}$. These results parallel previous findings ${ }^{7}$ and reiterate the possibility of insufficient sensitivity of ECG in detecting acute myocardial damage.

Table 5 Hazard ratios for all-cause mortality, adjusted for age and NIHSS score on admission

\begin{tabular}{llll}
\hline Characteristics & Hazard ratio & $\mathbf{9 5 \%} \mathrm{Cl}$ & p value \\
\hline cTnl $0.1-0.39 \mathrm{ng} / \mathrm{ml}$ & 2.14 & 1.13 to 4.05 & 0.01 \\
cTnl $\geqslant 0.4 \mathrm{ng} / \mathrm{ml}$ & 2.47 & 1.22 to 5.02 & 0.01 \\
Age, years & 1.05 & 1.02 to 1.08 & $<0.001$ \\
NIHSS score & 1.14 & 1.10 to 1.18 & 0.01 \\
\hline
\end{tabular}

NIHSS, National Institutes of Health Stroke Scale. 


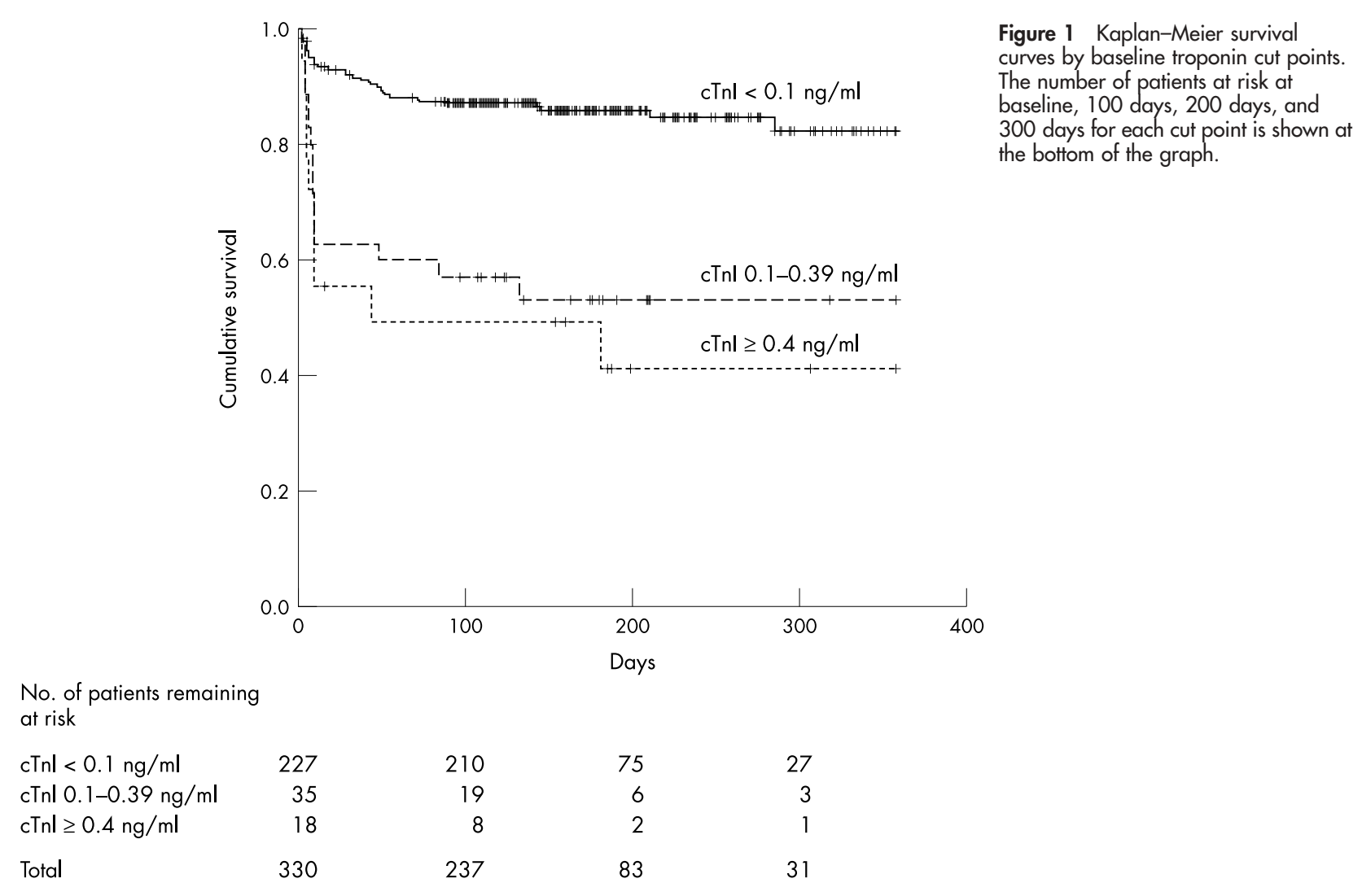

In our study, patients with acute ischaemic stroke with low or high abnormal cTnI levels on admission to an emergency department had an increased risk of death of 2.1 and 2.5, respectively, compared with patients with normal cTnI, an excess risk apparent across demographic and clinical subgroups. The fact that patients were accrued consecutively and prospectively and our choice of mortality as the main endpoint for the analysis reduced the possible bias which could have arisen from the partly retrospective retrieval of outcome data. Our results confirm the association between elevated troponin and poor outcome in patients with stroke, and extend previous findings, by also demonstrating higher mortality in patients with low troponin positivity. In addition, we have reported causes of death by troponin levels, which have not been previously reported in acute ischaemic stroke. Of the patients whose cTnI levels on admission to an emergency department were $0.4 \mathrm{ng} / \mathrm{ml}$ or higher, nearly half died in hospital. In that subgroup, roughly two thirds of patients died of cardiac causes, as opposed to one third cardiac deaths among patients with normal or low abnormal troponin. This difference did not reach statistical significance, but it seems reasonable to speculate that in a sizable proportion of patients with high positive troponin, myocardial damage either directly caused death or contributed significantly to an unfavourable outcome.

The excess mortality associated with low troponin positivity is more difficult to explain. Patients whose cTnI levels on admission were comprised (between $0.1 \mathrm{ng} / \mathrm{ml}$ and $0.39 \mathrm{ng} /$ $\mathrm{ml}$ ) did not die from a cardiac cause more often than patients with normal troponin. On the other hand, adjustment of stroke severity reduced but did not eliminate the significant association between low positive cTnI and an increased risk of death. Troponin levels on admission may therefore represent a surrogate marker for the systemic severity of acute ischaemic stroke, apparently able to exert a negative influence on the neurological evolution of the disease.
Another possible explanation is that troponin elevation may be a marker of unstable plaques in the coronary circulation, thereby indicating higher probability of an adverse prognosis due to recurrent myocardial ischaemia. In any case, it is worthwhile pointing out that in our cohort both low and high abnormal troponin levels retained their significant association with an increased risk of death even after adjustment of other well known systemic predictors of poor stroke outcome, such as glycaemia, ${ }^{17}$ fibrinogen, ${ }^{18}$ and white blood cell count. ${ }^{19}$

Our investigation has several limitations. First, in most cases we relied on a single baseline blood sample and thus we could not account for variations in troponin levels that occur over time. Repeat assays could provide additional information on the development and evolution of myocardial injury in patients with acute stroke and on its prognostic implications. Secondly, we did not investigate drug treatment as a possible confounder. Thirdly, we lacked data on the possible influence of the location of the brain lesion on the occurrence of the myocardial damage. In particular, involvement of the insular cortex is known to be associated with more frequent occurrence of cardiac complications after acute stroke, probably via an increased sympathetic tone mediated by the cerebral autonomic centres. ${ }^{20}$ The possibility that troponin elevations may be related to stroke involving the insula deserves further investigation.

Notwithstanding these limitations, these data extend current understanding of the implications of troponin positivity in acute ischaemic stroke. Clinicians should consider adding a cTnI test to the admission work up, as high troponin levels seem to impart a significantly higher risk of inhospital cardiac complications and death. CK-MB or myoglobin levels and ECG changes are much less accurate predictors. Rather than an indiscriminate strategy of systematic screening, it may be more cost efficient to limit the cTnI assay to patients having higher probability of a 
positive test-that is, those with a recent history of angina or MI, or those presenting with chest pain, ECG signs of cardiac ischaemia, or severe stroke. Whether further evaluation and treatment of cTnI positive patients can reduce cardiac morbidity and mortality should be the focus of future research.

\section{Authors' affiliations}

E Di Angelantonio, Department of Internal Medicine, University of Rome "La Sapienza", Rome, Italy

M Fiorelli, D Toni, M L Sacchetti, S Lorenzano, A Falcou, C Argentino, Department of Neurological Sciences, University of Rome "La

Sapienza", Rome, Italy

M Suppa, L Bonanni, G Bertazzoni, F Aguglia, Department of Emergency Medicine, University of Rome "La Sapienza", Rome, Italy M V Ciarla, Departments of Cellular Biotechnology and Hematology, University of Rome, Rome, Italy

This work was supported in part by a grant of the Italian Ministry of Health (ICS 030.6/RF00-49).

Competing interests: none declared

\section{REFERENCES}

1 Bounds JV, Wiebers DO, Whisnant JP, et al. Mechanisms and timing of deaths from cerebral infarction. Stroke 1981;12:474-7.

2 Silver FL, Norris JW, Lewis AJ, et al. Early mortality following stroke: a prospective review. Stroke 1984;15:492-6.

3 Johnston KC, Li JY, Lyden PD, et al. Medical and neurological complications of ischemic stroke: experience from the RANTTAS trial. RANTTAS Investigators. Stroke 1998;29:447-53.

4 Adams JE, Schechtman KB, Landt $Y$, et al. Comparable detection of acute myocardial infarction by $M B$ creatine kinase and cardiac troponin I. Clin Chem 1994;7:1291-5

5 Adams JE, Bodor GS, Davila-Roman VG, et al. Cardiac troponin I: a marker with high specificity for cardiac injury. Circulation 1993;88:101-6.
6 James $\mathbf{P}$, Ellis $\mathrm{CJ}$, Whitlock RM, et al. Relation between troponin $\mathrm{T}$ concentration and mortality in patients presenting with an acute stroke: observational study. BMJ 2000;320:1502-4

7 Dixit S, Castle M, Velu RP, et al. Cardiac involvement in patients with acute neurologic disease: confirmation with cardiac troponin I. Arch Intern Med 2000;160:3153-8

8 Brott T, Adams HP Jr, Olinger CP, et al. Measurements of acute cerebral infarction: a clinical examination scale. Stroke 1989;20:864-70.

9 Adams HP Jr, Bendixen BH, Kappelle $\amalg$, et al. Classification of subtype of acute ischemic stroke. Stroke 1993;24:35-41.

10 Hartmann A, Rundek T, Mast H, et al. Mortality and causes of death after first ischemic stroke. The Northern Manhattan Stroke Study. Neurology 2001;57:2000-5.

11 Myocardial infarction redefined-a consensus document of the Joint European Society of Cardiology/American College of Cardiology Committee for the redefinition of myocardial infarction. Eur Heart J 2000;21:1502-13.

12 Cannon CP, Battler A, Brindis RG, et al. American College of Cardiology key data elements and definitions for measuring the clinical management and outcomes of patients with acute coronary syndromes. A report of the American College of Cardiology Task Force on Clinical Data Standards (Acute Coronary Syndromes Writing Committee). J Am Coll Cardiol 2001;38:2114-30.

13 Morrow DA, Rifai N, Tanasijevic MJ, et al. Clinical efficacy of three assays for cardiac troponin I for risk stratification in acute coronary syndromes: a Thrombolysis In Myocardial Infarction (TIMI) 11B Substudy. Clin Chem 2000;46:453-60.

14 Morrow DA, Cannon CP, Rifai N, et al. Ability of minor elevations of troponins I and $\mathrm{T}$ to predict benefit from an early invasive strategy in patients with unstable angina and non-ST elevation myocardial infarction: results from a randomized trial. JAMA 2001;286:2405-12.

15 Norris JW, Hachinski VC, Myers MG, et al. Serum cardiac enzymes in stroke. Stroke 1979;10:548-53.

16 Kolin A, Norris JW. Myocardial damage from acute cerebral lesions. Stroke 1984;15:990-3.

17 Bruno A, Biller J, Adams HP Jr, et al. Acute blood glucose level and outcome from ischemic stroke. Trial of ORG 10172 in Acute Stroke Treatment (TOAST) Investigators. Neurology 1999;52:280-4.

18 Beamer NB, Coull BM, Clark WM, et al. Persistent inflammatory response in stroke survivors. Neurology 1998;50:1722-8.

19 Kasner SE, Demchuk AM, Berrouschot J, et al. Predictors of fatal brain edema in massive hemispheric ischemic stroke. Stroke 2001;32:2117-23.

20 Oppenheimer $\mathbf{S}$. The insular cortex and the pathophysiology of stroke-induced cardiac changes. Can J Neurol Sci 1992;19:208-11. 